Mol. Cryst. Liq. Cryst., 1993, Vol. 237, pp. 121-143

Reprints available directly from the publisher

Photocopying permitted by license only

(C) 1993 Gordon and Breach Science Publishers S.A.

Printed in the United States of America

\title{
Coupled Director and Layer Reorientation in Layer Tilted Ferroelectric Smectic Liquid Crystal Cells
}

\author{
FRANK GIEBELMANN and PETER ZUGENMAIER \\ Institut für Physikalische Chemie der Technischen Universität Clausthal, Arnold-Sommerfeld-Str. 4, \\ D-3392 Clausthal-Zellerfeld, Federal Republic of Germany
}

(Received April 6, 1992; in final form August 26, 1992)

A reversible reorientation of smectic layers in surface stabilized ferroelectric liquid crystal (SSFLC) cells with layer tilted (chevron) geometries was found by electrooptical investigations. The reorientation of the layers superimposes the reorientation of the director along the $\mathrm{S}_{\mathrm{C}}^{*}$ tilt cone and is suppressed, if the layers are irreversibly fixed in a bookself geometry by high electric fields.

Based on a potential density expansion, a dynamical model of a coupled director and layer reorientation is proposed, which leads to excellent agreement with the electrooptical behaviour of the system investigated. The fit of the model to experimental data supplies a simultaneous determination of several material and cell constants. Basic electrooptical parameters, as contrast, switching times and optical overshoot, are reproduced correctly.

Keywords: ferroelectric liquid crystals, SSFLC cells, switching dynamics, electrooptics, computer simulation

\section{INTRODUCTION}

In thin cells with unwound helical structures, the chiral smectic $\mathrm{C}^{*}$-phase exhibits macroscopic observable spontaneous polarization which depends on the position of the director relatively to the layer normal. ${ }^{1,2}$ An applied external field may change the position of the director through coupling. This coupling leads to the interesting electrooptical properties of the $\mathrm{S}_{\mathrm{C}}^{*}$-phase ${ }^{3}$ between crossed polars. It has been assumed that the director of the chiral smectic $C^{*}$ phase moves along the director tilt cone at constant tilt angle $\theta$ with the layer normal as cone axis.

Due to the size and the symmetry of surface interactions, ferroelectric liquid crystalline (FLC) cells generally exhibit a nonuniform splayed director distribution along the direction of the normal of the substrate plane without an electric field applied. These splayed cells are showing waveguiding effects and can not be brought into a position of complete extinction between crossed polars. If an electric field of sufficient strength is applied, all molecular directors reorient more or less along the tilt cone into the same position, caused by the interaction between the polarization and the electric field. This reorientation leads to an almost uniform director 
field. Consequently these uniform states act as uniform birefringent slab which can be brought into an extinction position between crossed polars. A detailed study of director orientations in surface stabilized FLC (SSFLC) cells is given by Maclennan et al. ${ }^{4}$

Computer simulations of the director reorientation in alternating electric fields based on the results in Reference 4 led to the conclusion that the switching process occurs via nonuniform splayed states. ${ }^{5,6}$ In contrast to these results we found strong experimental evidence for the switching process in the system used by us to be optically better described by a uniform director reorientation without any optically significant director distribution along the normal of the substrate. These experiments will be discussed in this paper.

It was shown in a previous investigation that electrooptical measurements in a SSFLC-cell allow the determination of the mean director motion during the switching process in the laboratory coordinate system, which is fixed to the substrates. ${ }^{7}$ The results in the laboratory coordinate system for the compound DOBAMBC in $2 \mu \mathrm{m}$ cells as well as the experiments presented in this paper lead to the conclusion that the director trajectory describes a slightly opened loop which connects the two stationary states with an almost straight line.

Assuming the director reorientation occurs uniformly without an optically observable distribution, then the observed mean director motion is more or less identical with the director motion of the uniformly oriented molecular directors in the bulk of the cell. The measured director trajectory seems to contradict the established model of director reorientation along the tilt cone, when the $\mathrm{S}_{\mathrm{C}}$-coordinate system defined by the tilt cone axis is fixed to the laboratory coordinate system. However, if a motion of the $S_{C}$-system relative to the laboratory system occurs during the switching process, the situation is changed. Taking the laboratory coordinate system as reference, the motion of the $S_{C}$-system superimposes the motion of the director within the $\mathrm{S}_{\mathrm{C}}$-system and consequently the observed trajectory differs from the circular segment of the pure tilt cone motion. ${ }^{8}$ A motion of the $\mathrm{S}_{\mathrm{C}}$-system is closely correlated with a reorientation of the smectic layers, because the $\mathrm{S}_{\mathrm{C}}$-system is defined by the tilt cone axis which represents the smectic layer normal.

\section{COORDINATE SYSTEMS AND LAYER CONFIGURATION}

In a previous paper $^{7}$ the layer normal case was presented, in which the laboratory coordinate system and the $\mathrm{S}_{\mathrm{C}}$-coordinate system are identical. It seems to be necessary for a description of the results presented in this paper to introduce the more general layer tilted geometry, including chevron geometry. Two separate coordinate systems have to be considered (Figure 1).

The laboratory coordinate system $x, y, z$ (cf. Figure 1) is defined by the optical axis of the microscope which lies along the $z$-axis. The external electric field as well as the normal of the glass plates of the FLC cell point in $z$-direction. The orthogonal coordinate system will completely be fixed by choosing the $y$-axis parallel to the intersection lines of the smectic layers with the glass plates. The elec- 


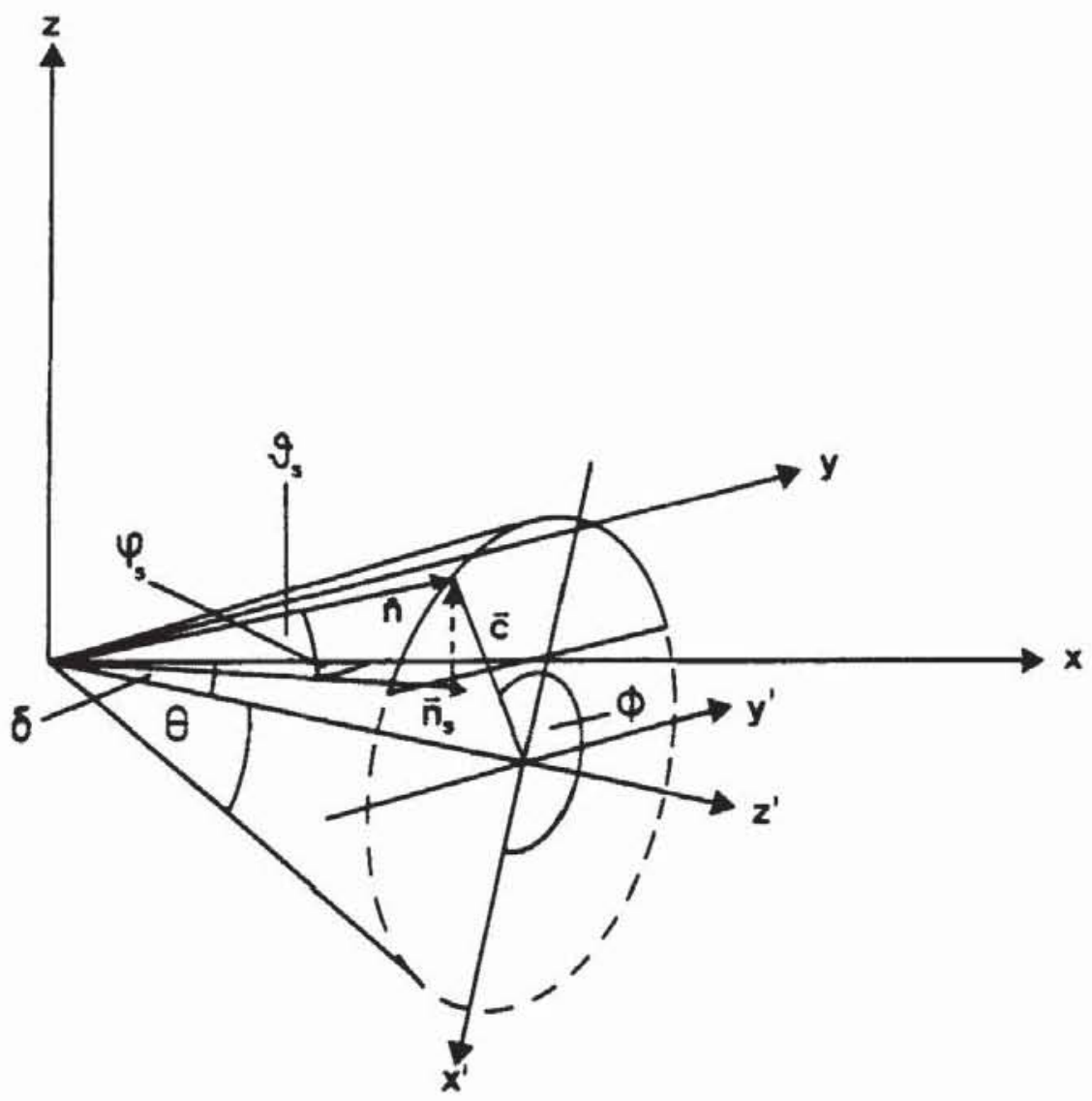

FIGURE 1 Coordinate systems for a description of director reorientation in FLC cells, $x, y, z$ is the laboratory coordinate system with copolar director coordinates $\vartheta_{s}$ (off-plane angle) and $\varphi_{s}$ (in-plane angle). $x^{\prime}, y^{\prime}, z^{\prime}$ is the $\mathrm{S}_{C}^{*}$ coordinate system with polar director coordinates $\theta$ (director tilt angle) and $\Phi$ (azimuth angle). $\delta$ is the smectic layer tilt angle, $\hat{z}$ the smectic layer normal, $\vec{c}$ the projection of the director $\hat{n}$ onto the smectic layer plane and $\hat{n}_{s}$ the projection of $\hat{n}$ onto the substrate plane.

trooptical experiment allows the determination of the copolar angle $\vartheta_{s}$ (with respect to the $z$-axis) and the azimuth angle $\varphi_{s}$ of the mean director during motion in this system. ${ }^{7}$ Generally, $\varphi_{s}$ describes the motion of the mean director in the plane of the glass plates and $\vartheta_{s}$ out of the plane. Therefore, these angles are called "inplane angle" $\varphi_{s}$ and "off-plane angle" $\vartheta_{s}$.

The orthogonal $\mathrm{S}_{\mathrm{C}^{-}}$-coordinate system $x^{\prime}, y^{\prime}, z^{\prime}$ (cf. Figure 1) is fixed by the position of the smectic layers in the $x^{\prime}, y^{\prime}$-plane $\left(y^{\prime}=y\right)$ and the layer normal $\hat{z}^{\prime}$ along $z^{\prime}$. The director position $\hat{n}^{\prime}$ will best be described in this coordinate system by the polar angle $\theta$, which is the director tilt angle of the $\mathrm{S}_{\mathrm{C}}^{*}$ phase, and the azimuth angle $\Phi$.

In case of a tilt of the smectic layers by an angle $\delta$ against the normal of the substrate $\hat{z}$, the $S_{\mathrm{C}}$-coordinate system will be obtained by a rotation of the laboratory coordinate system by $\delta-90^{\circ}$ around the $y$-axis. The director is represented in the laboratory coordinate system by:

$$
\hat{n}(x, y, z)=\left[\begin{array}{c}
\cos \vartheta_{s} \cos \varphi_{s} \\
\cos \vartheta_{s} \sin \varphi_{s} \\
\sin \vartheta_{s}
\end{array}\right]=\left[\begin{array}{c}
\sin \theta \cos \Phi \sin \delta+\cos \theta \cos \delta \\
\sin \theta \sin \Phi \\
\cos \theta \sin \delta-\sin \theta \cos \Phi \cos \delta
\end{array}\right]
$$


The director orientation in the laboratory coordinate system depends on the layer tilt angle $\delta$ and reversible or irreversible changes of the layer configuration during switching will affect the director trajectory and the transmittance response of the cell.

In recent papers of various authors irreversible changes of the orientation of smectic layers in layer tilted SSFLC-cells in electric fields have been reported. ${ }^{9,10}$ An irreversible reorientation of the smectic layers has been observed ${ }^{9}$ in strong electric fields corresponding to a rotation of the layer normal around the $y^{\prime}$-axis. Andersson et al. ${ }^{10}$ have found a reorientation of the smectic layers in the plane of the substrate corresponding to a rotation around the $x^{\prime}$-axis using asymmetric AC fields for the chiral $S_{A}$ phase.

Hartmann et al. ${ }^{11,12}$ have pointed out, that electrooptical effects observed in SSFLC-cells can be explained consistently, assuming a reversible, elastic layer reorientation. This idea is supported by Johno et al. ${ }^{13}$ Itoh et al. ${ }^{14}$ and Oh-E et al. ${ }^{15}$ who concluded from X-ray experiments a reversible layer reorientation. As will be presented here, reversible layer reorientation can directly be observed in the layer tilted case already at low electric field strength by electrooptical experiments. $^{8}$

\section{EXPERIMENTAL}

A detailed description of the experimental setup used for the electrooptical measurements is given in a previous paper. ${ }^{7}$ The experiments were carried out with a $4 \mu \mathrm{m}$ cell (E.H.C. Co. Ltd., Tokyo) filled with 4-[(S)-(+)-2-chloro-3-methyl-butyryloxy]-phenyl-4-decyloxy-benzoate ${ }^{16}$ (Aldrich Chem. Co., Steinheim). The measurements were performed $5^{\circ} \mathrm{C}$ below the $\mathrm{S}_{\mathrm{A}} \rightarrow \mathrm{S}_{\mathrm{C}}^{*}$ phase transition at 40.0 ${ }^{\circ} \mathrm{C}$.

The refractive indices, necessary for the evaluation of the data, have been provided by G. Pelzl, Institute for Physical Chemistry, MLU Halle-Wittenberg ( $n_{0}=$ $1.4842, n_{e}=1.6021$ at $40.0^{\circ} \mathrm{C}$ and $\left.\lambda_{0}=589 \mathrm{~nm}\right)$. The exact thickness of the cells was determined using the interference spectrum of the empty cells and applying the corrections given by Yang ${ }^{17}$ due to the optical influence of the polyimide and Ito layers.

\section{RESULTS AND DISCUSSION}

\subsection{Uniform and Nonuniform Director Reorientation}

Splayed or half splayed states are formed in the $4 \mu \mathrm{m}$ cells used in this investigation with a missing external electric field and are easily detectable since the intensity of light traveling through the cell cannot be extinguished completely between crossed polars. However, if a dc field with a sufficient field strength is applied, a uniform state appears and the light extinguishes between crossed polars. It is eminent for modelling the switching process to know whether the switching from one uniform state (e.g. polarization down) into the other (polarization up) occurs via splayed 
states and with a nonuniform director reorientation or if the director field remains uniformly and a uniform director reorientation has to be considered for a description of the switching. In the case of a nonuniform reorientation, the experimentally determined electrooptical behaviour has to be interpretated on the basis of continously changing director distributions while in the case of uniform reorientation the electrooptical behaviour can be described as reorientation of uniformly oriented molecular directors in the optically relevant volume of the cell. The following tests and considerations will provide evidence that, at least for the systems used in our experiments, the assumption of a uniform reorientation is justified.

(i) Optical uniformity during the switching process. According to Abdulalim, Moddel and Clark, the optical uniformity of the director field during switching may be probed experimentally by two observations ${ }^{6}$ :

- Extinction between crossed polars has to be obtained, when the director passes the polarization plane of the electric field vector of normally incident light.

- The extinction and its position in time during switching has to be independent on the wavelength of the incident light. This relates to the optical condition that the phase angle between the two optical modes $\left(\pi \cdot \Delta_{\text {opt }} / \lambda_{0} ; \Delta_{\text {opt }} ;\right.$ optical path difference) does not match a multiple of $\pi$.

Both conditions were checked experimentally for the system investigated in order to verify the assumption of optically uniform director reorientation. Figure 2 depicts a decay step of a $200 \mathrm{~Hz} / 1 \mathrm{MV} \mathrm{m}^{-1}$ electric square field, which was applied to a chevron layered cell of thickness $3.6 \mu \mathrm{m}$ and obtained after cooling from the $\mathrm{S}_{\mathrm{A}}$ phase. The corresponding transmission response between crossed polars at different cell orientations $\varphi_{0}$ and wavelengths of incident light $\lambda_{0}$ is shown in Figure 3 . The orientation of the cell relatively to the polarizer is represented by $\varphi_{0}$, which is the angle between the polarization plane of the incident light (electric field vector) and the projection of the smectic layer normal $\hat{z}^{\prime}$ onto the substrate plane. As it is shown in Figure 3(a), the director configuration at any point in time during switching can be set to extinction by variation of the cell orientation. This observation meets the first requirement cited above.

The second requirement was checked for several cell orientations at wavelengths $\lambda_{0}=633,589,546,520,492$ and $436 \mathrm{~nm}$ and some representative results are depicted in Figure 3(b). For any cell orientation $-\Theta \leqslant \varphi_{0} \leqslant+\theta$ and wavelengths

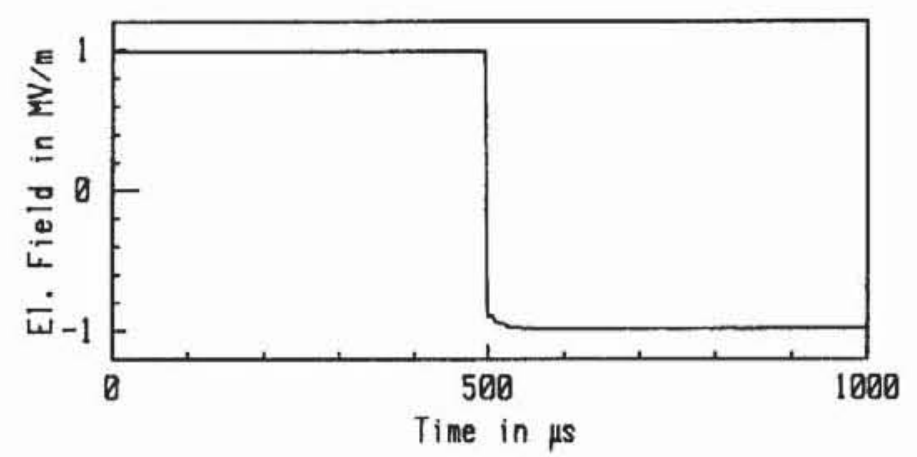

FIGURE 2 Decay step of a $200 \mathrm{~Hz} / 1 \mathrm{MV} \mathrm{m}^{-1}$ electric square field used for the measurements depicted in Figure 3. 

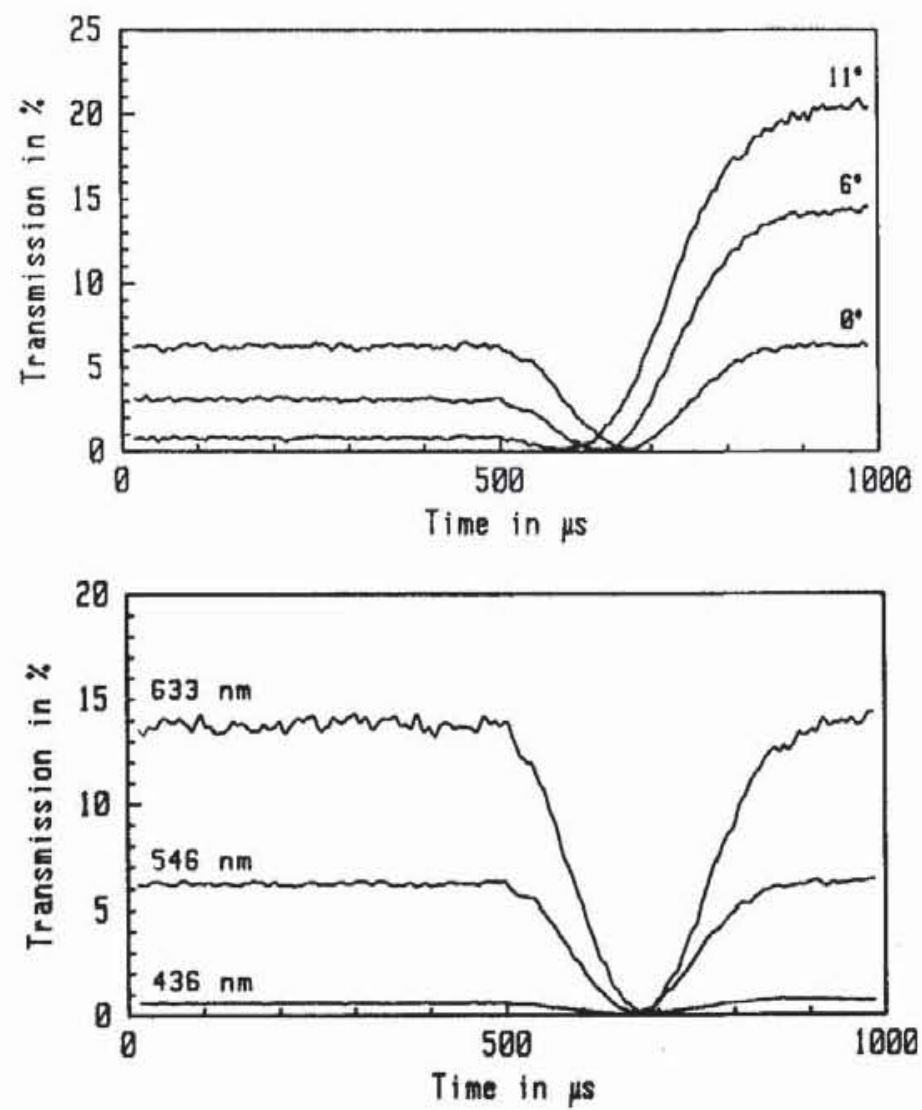

FIGURE 3 Transmittance response of the cell (thickness $3.6 \mu \mathrm{m}$ ) to the electric field depicted in Figure 2 at (a) various cell orientations $\varphi_{0}=0^{\circ}, 6^{\circ}$ and $11^{\circ}$ and wavelength $\lambda_{0}=546 \mathrm{~nm}$ and (b) various wavelengths $\lambda_{0}=633,546$ and $436 \mathrm{~nm}$ and cell orientation $\varphi_{0}=0^{\circ}$. Explanation see text.

$\lambda_{0}$ extinction was observed and the extinction point in time was not affected by the wavelength of light at fixed cell orientations.

From these observations it can clearly be concluded, that the director field remains optically uniform during switching and, therefore, the switching process may be treated optically as an uniform reorientation of the optical axis (optical uniform reorientation).

The experiments depicted in Figures 2 and 3 are essentially the same as those described by Abdulhalim et al. ${ }^{6}$ but they lead to completely different results for our system. Abdulhalim et al. observed transmission maxima instead of extinction at certain wavelengths and consequently concluded a reorientation of optically nonuniform director configurations.

ii) The dynamics of splayed state formation. Figure 4 shows the response of a $3.9 \mu \mathrm{m}$ cell at $40^{\circ} \mathrm{C}$ and $\lambda_{0}=589 \mathrm{~nm}$ to a $6 \mathrm{~Hz} / 4 \mathrm{~V}$ square voltage. No voltage is applied at the beginning of the frame (0-1 sec in Figure 4), and the nonuniform cell has a high transmission at $\lambda_{0}=589 \mathrm{~nm}$ due to the yellow appearance of the splayed state. Turning on the voltage, the cell switches between uniform up and down states (1-2 sec in Figure 4). During the switching procedures the transmission does not reach values, which come close to transmission of the nonuniform state. After switching off the field, the cell requires more than $2 \mathrm{sec}$ to transform into the nonuniform splayed state again (2.0-4.5 sec in Figure 4). 

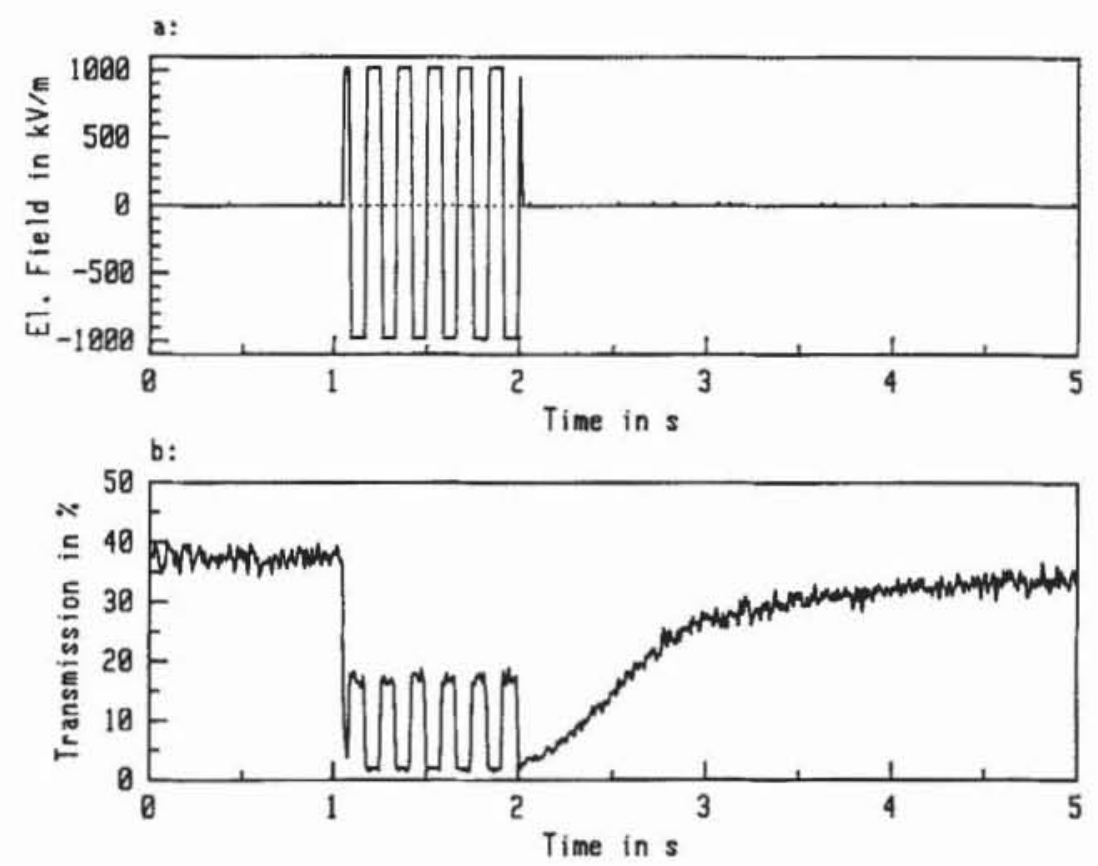

FIGURE 4 Relaxation from uniform states into the nonuniform state for a (a) $6 \mathrm{~Hz} / 4 \mathrm{~V}$ square voltage and (b) corresponding transmission. Explanation see text.

Since the relaxation of the system takes about 2 seconds, it seems impossible to form a splayed state in a few milliseconds during the switching procedure. In the actually performed experiments $200 \mathrm{~Hz}$ have been used instead of $6 \mathrm{~Hz}$ as in Figure 4.

In conclusion it should be clearly stated that the experimentally proven fact of an optically uniform describable switching behaviour does not mean, that nonuniformities will not exist in the cells rather that they will not be observed in our experiments. From all what is known today about switching dynamics in SSFLC cells, it is quite clear that there is no reorientation of a completely uniform director field in these cells. Furthermore, the splay elastic energy which is connected to nonuniform director fields essentially affects the switching dynamics and no realistic description of SSFLC switching had been achieved without considering splay elastic energy. On the other hand, simulations of nonuniform director reorientation ${ }^{5.6}$ indicate, that the splay deformation is not distributed linearly along the cell during switching rather that the splay is localized within more or less thin sections of the cell, which are denoted as "kinks" in the paper of Abdulhalim et al. ${ }^{6}$ Therefore, the director configuration of the cell may be approximated by a sequence of uniform and nonuniform sections (see Section 4.3).

In order to describe the transmission of the cell during switching the key question has to be clarified, whether the dimensions of the nonuniform sections are sufficient to dominate the optics of the cell at the wavelength considered, or not. In the latter case the optical properties are mainly determined by the director orientation of the uniform sections and the director reorientation appears optically as a uniform reorientation. Consequently, the transmission behaviour during switching serves as a probe for the director reorientation dynamics in the uniform sections of the cell and may be analyzed by the method proposed in Reference 7. 
Optically uniform switching is expected, if the polarization coherence length $\xi_{p}$

$$
\xi_{p}=\sqrt{4 \pi \varepsilon_{0} K_{\text {splay }} / P_{0}^{2}}
$$

is small compared to both, the light wavelength $\lambda_{0}$ and the cell thickness $d$. With values $P_{0}=1.9 \cdot 10^{-3} \mathrm{C} \mathrm{m}^{-2}$ and $K_{\text {splay }}=160 \mathrm{pN}$ for the system investigated, a coherence length $\xi_{p} \approx 70 \mathrm{~nm}$ results which is indeed very small compared to the wavelength $\lambda_{0}=546 \mathrm{~nm}$ and the cell thickness $d \approx 4 \mu \mathrm{m}$ used in our experiments. Therefore uniform switching behaviour should be expected as it is actually observed. The system investigated by Abdulhalim et al. exhibits far less spontaneous polarization and leads to $\xi_{p} \approx 533 \mathrm{~nm}$ which matches the wavelength $(524 \mathrm{~nm})$ at which they observed strongly nonuniform optical behaviour.

\subsection{Coupled Director and Layer Reorientation}

The electrooptical measurements resulting in the mean director motion depicted in Figure 5 have been performed with a $3.9 \mu \mathrm{m}$ cell using a square wave voltage of $200 \mathrm{~Hz}$ and $3.9 \mathrm{~V}$ amplitude at an optical wavelength of $\lambda=546 \mathrm{~nm}$. The amplitude of the voltage was increased between the individual measurements for a period of $1 \mathrm{sec}$ to $39 \mathrm{~V}$. This electrical high field treatment led to a significant change of the transmission curves and the resulting director motion when applied for some time.

A representative series of measurements is depicted in Figure 5 in which the director orientations determined at the switching process are represented as tra-
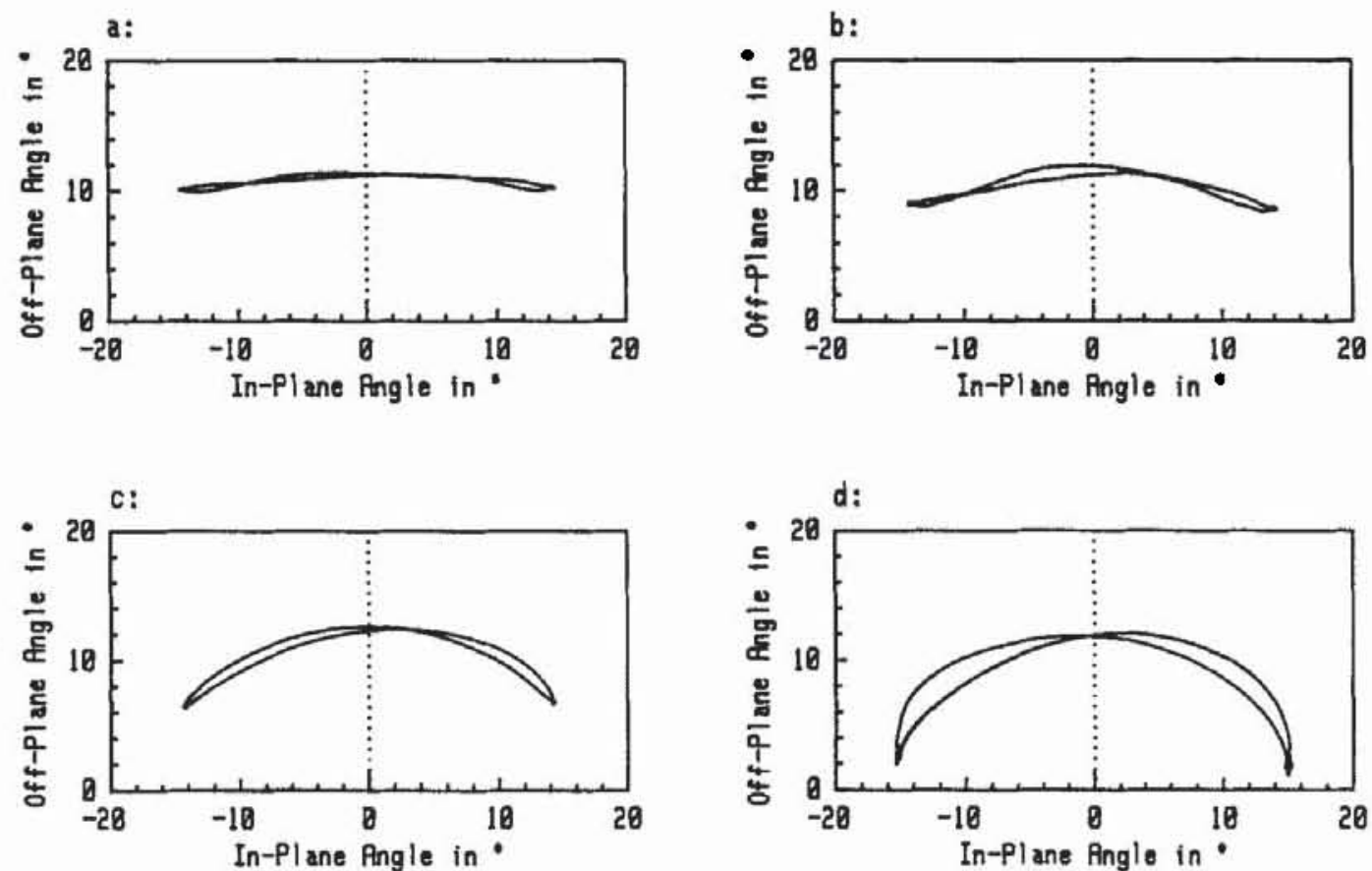

FIGURE 5 Trajectories of the mean director reorientation in a $200 \mathrm{~Hz}, 1 \mathrm{MV} \mathrm{m}^{-1}$ electric square field measured with a $3.9 \mu \mathrm{m}$ cell $\left(40^{\circ} \mathrm{C}\right)$ and after different duration of electrical high field treatment (HFT) (a) $0 \mathrm{sec}$, (b) $2 \mathrm{sec}$, (c) $5 \mathrm{sec}$ and (d) $20 \mathrm{sec}$ of total time of electrical high field treatment. 
jectories on the unit sphere in the laboratory coordinate system. The plot of the off-plane angle $\vartheta_{s}$ versus the inplane angle $\varphi_{s}$ approximately corresponds to a view in $-x$ direction of the laboratory coordinate system onto the $y, z$-plane (cf. Figure 1) at least at small angles.

Figure 5(a) shows the trajectory of director reorientation resulting from the first measurement at a virgin cell. Both stationary switching states of the $\mathrm{S}_{\mathrm{C}}^{*}$ phase are connected by an almost straight line. The motion of the director in the plane of the glass plates is large with ca. $30^{\circ}$ as compared to the size of the motion out of the plane of the glass plates with $\vartheta_{s}=10-12^{\circ}$. In the laboratory coordinate system no hints for a reorientation along the tilt cone are found which would lead to slightly distorted segments of circles in the $\varphi_{s}, \vartheta_{s}$-plot at fixed layers (cf. Figure 1). The diagram qualitatively corresponds the ones found for the compound DOBAMBC. ${ }^{7}$

Figure 5(d) represents the director motion in the same cell after a total of 20 $\mathrm{sec}$ high field treatment in intervals of $1 \mathrm{sec}$. Now the director nearly describes the expected segment of a circle and may be interpretated as a pure, uniform reorientation of the director along the $\mathrm{S}_{\mathrm{C}}^{*}$ tilt cone which agrees with the predicted director motion for a perpendicular bookshelf geometry. ${ }^{18}$ Intermediate states during the high field treatment are plotted in Figures 5(b) and 5(c) after 2 and $5 \mathrm{sec}$ high field application, respectively. The original motion of the director (Figure 5(a)) seems to proceed towards the reorientation along the tilt cone (Figure 5(d)) as can be concluded from these two figures.

Polarization microscopic observations at different stages of this treatment reveal that the transition from layer tilted to bookshelf geometry induced by the high field treatment will not be passed homogeneously: The cell shows a homogeneous, almost defect free yellow texture before the high field treatment, and a similar, yet red texture after completion of the treatment. At intermediate stages of the high field treatment, the texture appears strongly inhomogeneous: Yellow and red domains are observed side by side as well as numerous defects, especially zig-zag lines. The red domains grow increasingly larger as the high field treatment proceeds. The defects disappear upon completion of the high field treatment and the cell exhibits a homogeneous red colour in the polarizing microscope.

The experimental results suggest that the tilted smectic layers at the beginning of the experiment will irreversibly be raised by the high electric field until the layer normal case is reached. In the latter case the director motion in the laboratory coordinate system follows the uniform reorientation of the director along the $\mathrm{S}_{\mathrm{C}}^{*}$ tilt cone. If the layers are tilted, however, the torque on the layers, even at small field strength, produces a reversible reorientation of the layers during the switching process. The reorientation of the director along the tilt cone will additionally be superimposed by the reversible reorientation of the layers.

The proposed mechanism of the electrooptical switching process in an idealized layer-tilted FLC-cell is illustrated schematically in Figure 6. Different situations during the switching process from one stationary state $(a)$ to the other $(e)$ are depicted. During the zero transition of the applied square wave field, the cell exhibits a layer tilted structure, corresponding to Figure 6(c). The layer normal $\hat{z}^{\prime}$, represented by the tilt cone axis, is placed at a certain angle $\delta_{0}$ to the substrate ( $x, y$-plane), which is the layer tilt angle (cf. Figures 1 and $6(c))$. The azimuth 


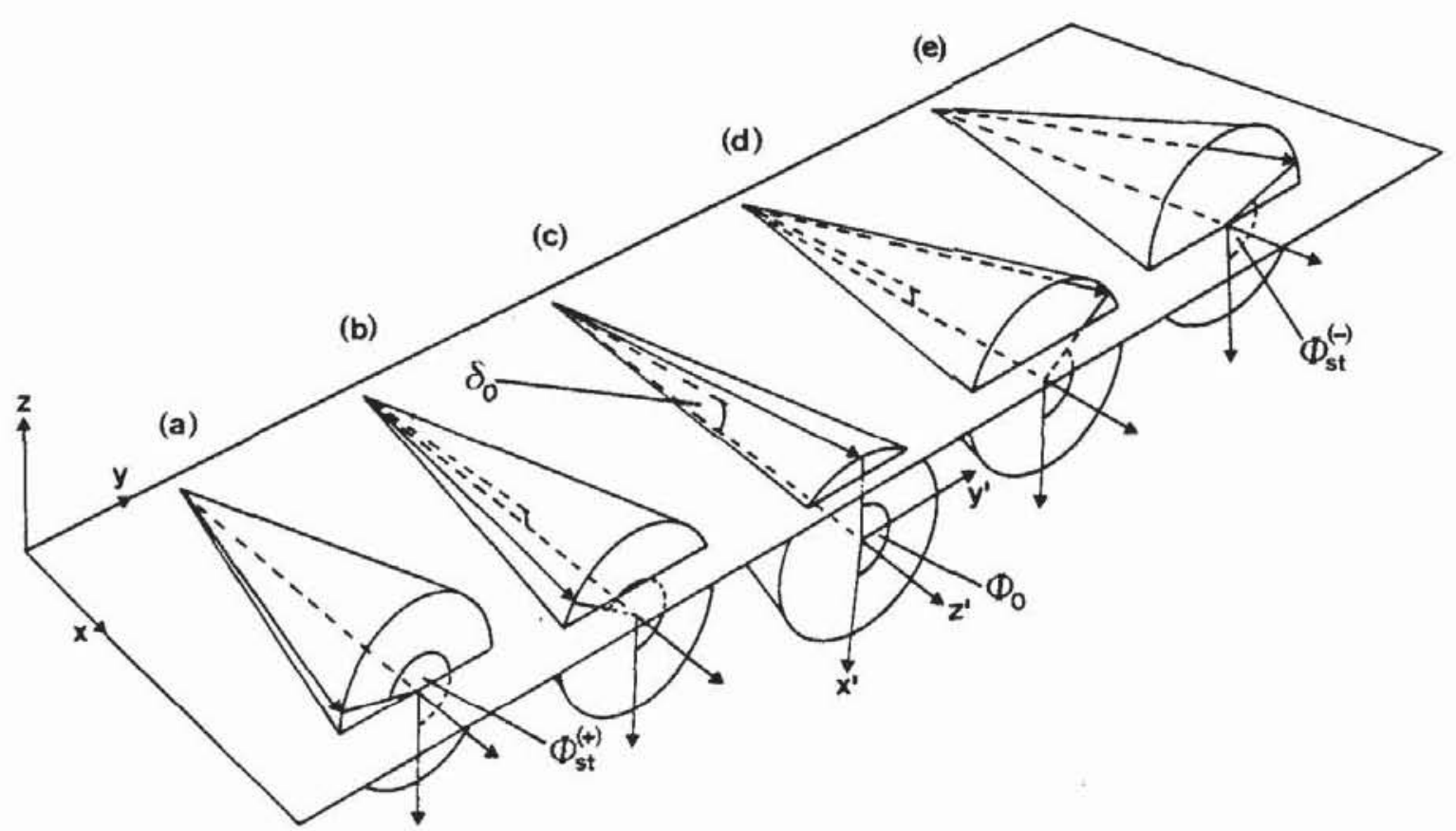

FIGURE 6 Schematic representation of a coupled director and layer reorientation. Explanation see text.

angle also has an equilibrium value $\Phi_{0}$ (cf. Figure 3(c)), depending on the anchoring conditions.

If the electric field strength increases, the cell finally reaches a stationary state, depicted in Figure 6(a). During the transition from $c$ to $a$ the director $\hat{n}$ rotates round $\hat{z}^{\prime}$ along the tilt cone surface, changing the azimuth angle from $\Phi_{0}$ (Figure 6(c)) to $\Phi_{s t}^{(+)}$(Figure 6(a)) and simultaneously the layer tilt angle decreases from $\delta=\delta_{0}$ to $\delta \approx 0$ (Figure $6(\mathrm{a})$ ). An inversion of the external field direction leads to a motion in reverse sequence via the layer tilted state (Figure 6(c)) into the second stationary state (Figure 6(e)) with $\Phi=\Phi_{s t}^{(-)}$and $\delta \approx 0$. Figures $6(\mathrm{~b})$ and 6(d) indicate some intermediate states during the transition.

The reorientation of the director in $\Phi$ along the surface of the $S_{C}^{*}$ tilt cone is superimposed by the layer reorientation, represented by the motion of the cone axis in $\delta$. In the laboratory coordinate system $x, y, z$ this superposition leads, as shown in Figure 6, to a director motion along a straight line (note the constant distance between the director and the $x, y$-plane in Figures 6(a)-6(e) from one stationary state to the other or to the depicted trajectory in Figure 5(a).

\subsection{Modelling the Coupled Director and Layer Reorientation}

A model for the SSFLC cell depicted in Figure 7 was developed which predicts the experimental data of the previous chapter. The model approximates the director configuration of the cell by a sequence of various uniform and nonuniform sections with the following assumptions:

(i) The cell exhibits a chevron layered structure. The center plane $c i$ of the chevron interface represents a local mirror plane at which the azimuth angle of the director is fixed with $+\pi / 2$ and $-\pi / 2$, respectively. 


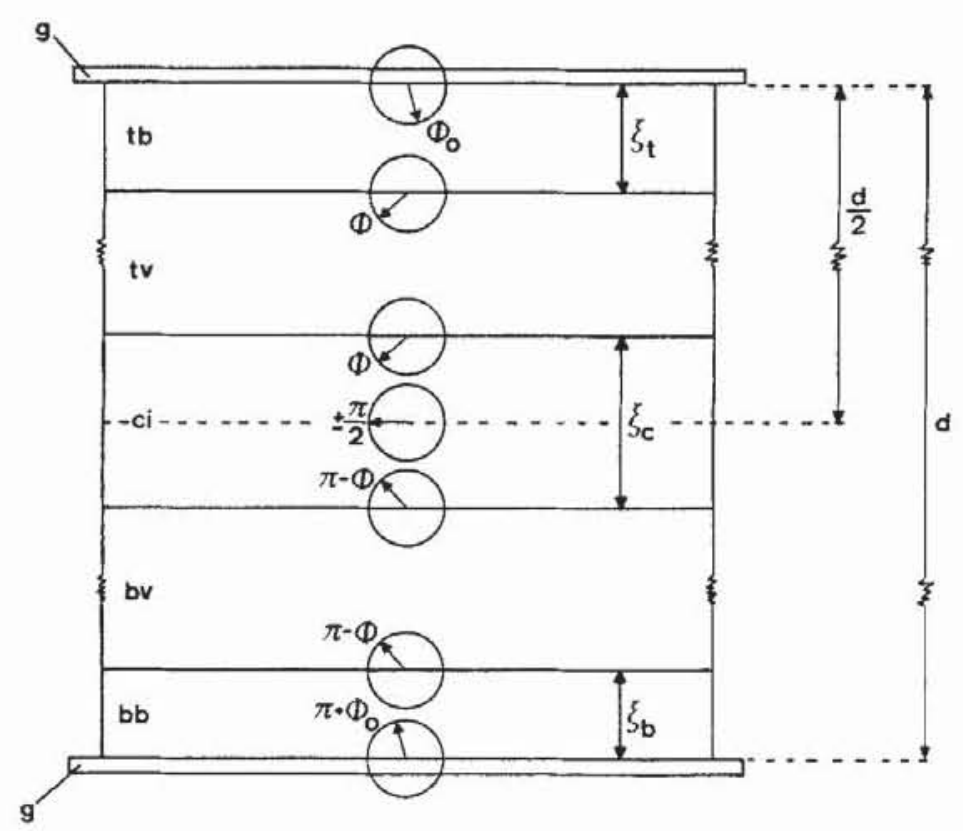

FIGURE 7 Model of an SSFLC cell under an applied electric field. The cell is divided into several sections. Uniform sections are the top $(t v)$ and bottom $(b v)$ volume layer. Nonuniform sections are the top $(t b)$ and the bottom $(b b)$ boundary layer with thickness $\xi_{t}$ and $\xi_{b}$, respectively, and the chevron interface $(\mathrm{ci})$ with thickness $\xi_{c}$. The circles with $\vec{c}$ directors inside indicate the director distribution across the cell with thickness $d$. $\Phi_{0}$ is the azimuth angle of the director at the top glass plate $(g)$ and $\Phi$ the azimuth angle in the top volume layer.

(ii) The boundary conditions at the upper as compared to the lower substrate surface are related by $\mathrm{C}_{2}$-symmetry (parallel rubbed surfaces). If the $\vec{c}$ director is anchored at an angle $\Phi_{0}$ to the upper substrate surface, the angle amounts to $\pi+\Phi_{0}$ at the lower substrate surface. The total splay of the $\vec{c}$-director is constant and amounts to $\pi$ across the cell thickness $d$.

(iii) The SSFLC-cell is devided into several layers of different thickness: top and bottom boundary layers $\xi_{t}, \xi_{b}$, the chevron interface $\xi_{c}$ and large volume sections in between, in which a uniform director configuration is assumed, supposed the cell is placed in a sufficient large electric field.

(iv) The layer thickness $\xi_{t}, \xi_{b}, \xi_{c}$ are changing during the switching procedure and hindered in their motion by a viscous damping. These assumptions allow the description of the slow transformation of an optically uniform director configuration with $\xi_{t}+\xi_{b}+\xi_{c} \ll d$ into an optically nonuniform director configuration with $\xi_{t}+\xi_{b}+\xi_{c} \approx d$.

The optical properties of the cell are determined by the predominant portion of the cell volume. This volume is represented by the uniform sections $t v, b v$ in cases when the $\vec{P}, \vec{E}$ interaction surpasses the elastic forces and the nonuniform sections $t b, c i, b b$ remain small. The splay elastic energy is predominantly localized in the nonuniform layers $t b, c i, b b$.

The proposed model leads then to the following expansion of the potential density of the cell 


$$
\begin{aligned}
g= & g_{0}+\sum_{i=1}^{3} \frac{a_{i}}{2 i} \Theta^{2 i}+\frac{K_{\text {splay }} \Theta^{2} \cos ^{2} \delta}{2 d}\left[\frac{\left(\Phi_{0}-\Phi\right)^{2}}{\xi_{t}}+\frac{(\pi-2|\Phi|)^{2}}{\xi_{c}}+\frac{\left(\Phi_{0}+\Phi\right)^{2}}{\xi_{b}}\right] \\
+ & \frac{K_{\text {pre }}}{2}\left(\vartheta_{0}-\delta+\Theta \cos \Phi\right)^{2}-\left(1-\frac{\xi_{t}+\xi_{c}+\xi_{b}}{d}\right)\left[P_{0} E \sin \Theta \cos \delta \sin \Phi\right. \\
& \left.+\frac{\Delta \varepsilon}{2} E^{2}\left(\sin ^{2} \Theta \cos ^{2} \delta \cos ^{2} \Phi+\cos ^{2} \Theta \sin ^{2} \delta-\frac{1}{2} 2 \theta \sin 2 \delta \cos \Phi\right)\right]
\end{aligned}
$$

and will now be discussed.

Generally, the contribution of the splay deformation to the potential density is given by:

$$
g_{\text {splay }}=\frac{1}{2} K_{\text {splay }} \Theta^{2}(\nabla \Phi)^{2}
$$

If a linear director distribution across the sections of the cell is assumed, the splay elastic energy density of the several layers amounts to:

top boundary layer $(t b)$ :

$$
g_{\text {splay }, t b}=\frac{1}{2} K_{\text {splay }} \Theta^{2} \cos ^{2} \delta \frac{\left(\Phi_{0}-\Phi\right)^{2}}{\xi_{t}^{2}}
$$

top volume layer $(t v)$ :

$$
g_{\text {splay }, t v}=0
$$

chevron interface $(c i)$ :

$$
g_{\text {splay } . c i}=\frac{1}{2} K_{\text {splay }} \Theta^{2} \cos ^{2} \delta \frac{(\pi-2|\Phi|)^{2}}{\xi_{c}^{2}}
$$

bottom volume layer $(b v)$ :

$$
g_{\text {splay }, b v}=0
$$

bottom boundary layer $(b b)$ :

$$
g_{\text {splay }, b b}=\frac{1}{2} K_{\text {splay }} \theta^{2} \cos ^{2} \delta \frac{\left(\Phi_{0}+\Phi\right)^{2}}{\xi_{b}^{2}}
$$

with $K_{\text {splay }}$ elastic splay constant, $\Phi_{0}$ azimuth angle of the director at the top boundary surface and $\Phi$ azimuth angle of the director in the uniform top volume layer. 
The contribution of the splay deformation to the potential density of the total cell has to be calculated by integration across the cell thickness $d$ and is given by:

$$
\begin{aligned}
g_{\text {splay }} & =\frac{\int_{0}^{d} g_{\text {play }, i} d x}{\int_{0}^{d} d x}=\frac{1}{d}\left(\int_{0}^{\xi_{t}} g_{\text {splay }, t b} d x+\int_{0}^{\xi_{c}} g_{\text {splay }, c i} d x+\int_{0}^{\xi_{b}} g_{\text {splay }, b b} d x\right) \\
& =\frac{K_{\text {splay }} \Theta^{2} \cos ^{2} \delta}{2 d}\left[\frac{\left(\Phi_{0}-\Phi\right)^{2}}{\xi_{t}}+\frac{(\pi-2|\Phi|)^{2}}{\xi_{c}}+\frac{\left(\Phi_{0}+\Phi\right)^{2}}{\xi_{b}}\right]
\end{aligned}
$$

The interaction between the molecules of the liquid crystal and the surface of the glass boundary results in an initial azimuth angle $\Phi_{0}$ and an off-plane angle $\vartheta_{0}$ of the director, the so called pretilt angle between the director $\hat{n}$ and the substrate surface. It should be noted, that this definition deviates to some extent from the one of Takezoe, Ouchi, Ishikawa and Fukuda. ${ }^{19} \mathrm{~A}$ deviation from the pretilt angle $\vartheta_{0}$ gives rise to a deformation energy $g_{\text {pre }}$ which is considered in a harmonic approximation with $\vartheta \approx \delta-\Theta \cos \Phi$ (cf. Figure 1):

$$
g_{\text {pre }}=\frac{1}{2} K_{\text {pre }}\left(\vartheta_{0}-\vartheta\right)^{2}=\frac{1}{2} K_{\text {pre }}\left(\vartheta_{0}-\delta+\Theta \cos \Phi\right)^{2}
$$

The pretilt constant $K_{\text {pre }}$ is an effective elastic constant which describes the anchoring strength of the molecules at the boundary surface. It will be changed by a preconditioning of the cell.

The dielectric displacement $\vec{D}$ of the FLC is composed of the spontaneous polarization $\vec{P}_{s}$ and the induced polarization $\vec{P}_{\text {ind }}$ :

$$
\begin{aligned}
\vec{D} & =\varepsilon_{0} \vec{E}+\vec{P}_{s}(\Theta, \Phi, \delta)+\vec{P}_{\text {ind }}(\Theta, \Phi, \delta, \vec{E}) \\
& =\varepsilon_{0} \vec{E}+P_{0}[\hat{z} \times \hat{n}]+\varepsilon(\Theta, \Phi, \delta) \vec{E}
\end{aligned}
$$

The $\varepsilon$ tensor does not describe the effective permittivity of the ferroelectric material rather then is related to the polarization which will be induced without spontaneous polarization of the compound but exhibiting the same dielectric properties (cf. Dahl et al. ${ }^{20}$ ). The most important contribution to the $\vec{P}, \vec{E}$ interaction results from the uniform sections ( $t v$ and $b v$ in Figure 7) in which the polarization is directed uniformly and optimal to the electric field. This is not the case in the nonuniform sections $(t b, c i, b b)$, because of the splayed director configuration and a compensation in part between the various nonuniform layers across the cell, and their contributions will not be considered in a first approximation.

The energy density of the spontaneous polarization in the applied electric field $E$ can be calculated to:

top volume layer $(t v)$ :

$$
g_{P_{s}-E, t v}=-P_{0} E \sin \Theta \cos \delta \sin \Phi
$$


bottom volume layer $(b v)$ :

$$
\begin{aligned}
g_{P_{s}-E, b v} & =-P_{0} E \sin \theta \cos \delta \sin (\pi-\Phi) \\
& =-P_{0} E \sin \theta \cos \delta \sin \Phi
\end{aligned}
$$

which leads to a total of:

$$
g_{P_{s}-E}=-\left(1-\frac{\xi_{t}+\xi_{c}+\xi_{b}}{d}\right) P_{0} E \sin \theta \cos \delta \sin \Phi
$$

The induced polarization with $\varepsilon=\varepsilon_{\perp}$ and $\Delta \varepsilon=\varepsilon_{\|}-\varepsilon_{\perp}$ contributes similarily, not considering the biaxiality of the $S_{C}^{*}$ phase:

$$
\begin{aligned}
g_{P_{\text {ind }}-E}=- & \left(1-\frac{\xi_{t}+\xi_{c}+\xi_{b}}{d}\right) \frac{\Delta \varepsilon E^{2}}{2}\left(\sin ^{2} \Theta \cos ^{2} \delta \cos ^{2} \Phi\right. \\
& \left.+\cos ^{2} \Theta \sin ^{2} \delta-\frac{1}{2} \sin 2 \theta \sin 2 \delta \cos \Phi\right)-\frac{1}{2} \varepsilon E^{2}
\end{aligned}
$$

The last term to be considered with a contribution to the total potential density of Equation (2) represents a simple Landau expansion in $\theta$ :

$$
g_{\text {Landau }}=\sum_{i=1}^{3} \frac{a_{i}}{2 i} \Theta^{2 i}
$$

Evaluation of Equation (2) leads to the Equations of motion (12a-f) as function of the internal coordinates $\Theta, \Phi, \delta, \xi_{t}, \xi_{c}, \xi_{b}$ and their solution to a dynamic model.

$$
\begin{aligned}
\gamma_{\Theta} \frac{\partial \Theta}{\partial t} & =-\left(\frac{\partial g}{\partial \Theta}\right)_{\Phi, \delta, \xi_{t}, \xi_{c}, \xi_{b}} \\
\gamma_{\Phi} \sin ^{2} \Theta \frac{\partial \Phi}{\partial t} & =-\left(\frac{\partial g}{\partial \Phi}\right)_{\Theta, \delta, \xi_{t}, \xi_{c}, \xi_{b}} \\
\gamma_{\delta} \frac{\partial \delta}{\partial t} & =-\left(\frac{\partial g}{\partial \delta}\right)_{\Theta, \Phi, \xi_{t}, \xi_{c}, \xi_{b}} \\
\gamma_{\xi} \frac{\partial \xi_{t}}{\partial t} & =-\left(\frac{\partial g}{\partial \xi_{t}}\right)_{\Theta, \Phi, \delta, \xi_{c}, \xi_{b}} \\
\gamma_{\xi} \frac{\partial \xi_{c}}{\partial t} & =-\left(\frac{\partial g}{\partial \xi_{c}}\right)_{\Theta, \Phi, \delta, \xi_{t}, \xi_{b}} \\
\gamma_{\xi} \frac{\partial \xi_{b}}{\partial t} & =-\left(\frac{\partial g}{\partial \xi_{b}}\right)_{\Theta, \Phi, \AA, \xi_{t}, \xi_{c}}
\end{aligned}
$$


$\gamma_{\Theta}, \gamma_{\Phi}, \gamma_{\delta}, \gamma_{\xi}$ represent viscous damping constants for the motion of the director in $\theta, \Phi$, the motion of the layers $\delta$ and the splay deformation $\xi$ (in $\xi_{t}, \xi_{c}, \xi_{b}$ ), respectively. All motions are considered as free of inertia.

Solving the set of 6 coupled differential Equations $(12 \mathrm{a}-\mathrm{f})$ leads to the motion of the director in the uniform sections $t v$ and $b v$ (Figure 7) in $\Phi$ and $\theta$, a possible motion of layers in $\delta$ and to a change of thickness of the nonuniform sections $t b$, $c i$ and $b b$ (Figure 7) in $\xi_{t}, \xi_{c}$ and $\xi_{b}$ during the switching procedure. The set of differential equations is solved numerically with a 4th order Runge-Kutta algorithm for the shape of the experimentally applied field $E(t)$.

The functions $\Phi(t), \Theta(t)$ and $\delta(t)$ obtained are converted to the laboratory coordinate system by (cf. Equation 1):

$$
\begin{aligned}
& \sin \vartheta_{s}=\cos \theta \sin \delta-\sin \theta \cos \Phi \cos \delta \\
& \tan \varphi_{s}=\frac{\sin \theta \sin \Phi}{\sin \theta \cos \Phi \sin \delta+\cos \theta \cos \delta}
\end{aligned}
$$

and compared to experimentally determined trajectories of the director. Variation of the cell and material constants leads to a best fit of theoretically calculated towards experimental data.

Stationary solutions for the set of differential equations describe the determined trajectories before (Figure 5(a)) and after (Figure 5(d)) the high field treatment and are depicted in Figures 8(a) and 8(b), respectively. The parameters from the fitting procedure are listed in Table I and will be discussed later. A comparison of Figure 5(a) with Figure 8(a) and Figure 5(d) with Figure 8(b) shows, that the observed trajectories are nicely described by the proposed dynamic model. Before the high field treatment, a compensation of the off-plane angles occurs by a reorientation of the layers leading to little dependency in the off-plane angle in the laboratory coordinate system (Figures 5(a) and 8(a)). A reorientation of the director along the tilt cone is the only observed motion in bookshelf geometry and results in the expected trajectories (Figures 5(d) and 8(b)).

A more direct comparison with experimental data results, if the transmission curves $T(t)$ resulting from the theoretically obtained director trajectory $\varphi_{s}(t), \vartheta_{s}(t)$ are calculated, assuming an almost uniform director configuration by

$$
T=\sin ^{2}\left[2\left(\varphi+\varphi_{0}\right)\right] \sin ^{2}\left(\frac{\pi d \Delta n\left(\vartheta_{s}\right)}{\lambda_{0}}\right)
$$
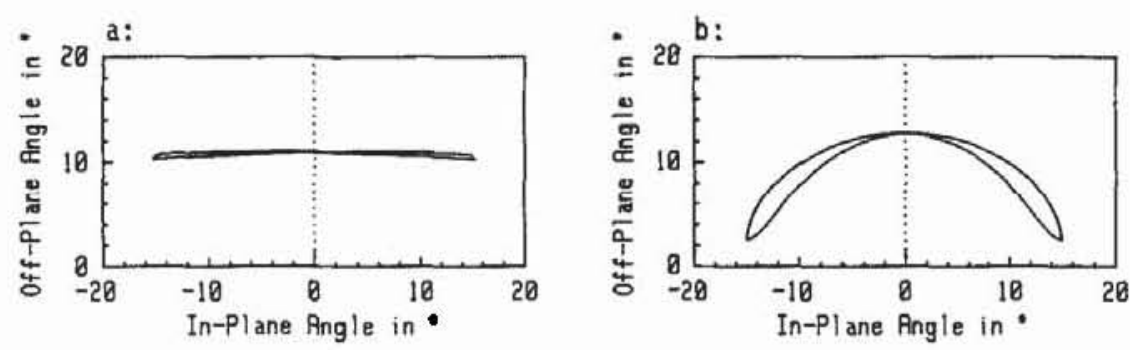

FIGURE 8 Trajectories of the mean director reorientation in a $200 \mathrm{~Hz}, 1 \mathrm{MV} \mathrm{m}^{-1}$ electrical square field calculated from the proposed model (a) before and (b) after completed high field treatment. (a) corresponds to Figure 5(a) and (b) to Figure 5(d). 


\section{TABLE I}

Material and cell constants obtained from a simulation of the electrooptical behaviour before $(0 \mathrm{sec}$ HFT) and after completed ( $20 \mathrm{sec}$ HFT) high field treatment

\begin{tabular}{|c|c|c|c|}
\hline Constant & $0 \mathrm{sec} H F T$ & $20 \mathrm{sec} H F T$ & Remarks \\
\hline $\begin{array}{l}\text { Landau Constants: } \\
\qquad \begin{array}{r}a_{1} / \mathrm{kN} \mathrm{m}^{-2} \\
a_{2} / \mathrm{kN} \mathrm{m}^{-2} \\
a_{3} / \mathrm{MN} \mathrm{m}^{-2}\end{array}\end{array}$ & & & $\begin{array}{l}\text { Landau constants give } \theta_{0}=13.8^{\circ} \\
\text { X-ray measurement: } \theta_{0}=14^{\circ}\end{array}$ \\
\hline $\begin{array}{l}\text { Elastic Properties: } \\
\qquad \begin{array}{c}\Phi_{0} / \text { degree } \\
K_{\text {splay }} / p N \\
\vartheta_{0} / \text { degree } \\
K_{\text {pre }} / \mathrm{kN} \mathrm{m}^{-2}\end{array}\end{array}$ & 20 & 0 & $\begin{array}{l}\mathrm{K}_{\text {eff }}=\theta^{2} \mathrm{~K}_{\text {splay }}=9.1 \mathrm{pN} \\
5 \mathrm{pN}<\mathrm{K}_{\text {eff }}<50 \mathrm{pN}\end{array}$ \\
\hline $\begin{array}{l}\text { Viscosities: } \\
\qquad \begin{array}{c}\gamma_{\Phi} / \mathrm{Pas} \\
\gamma_{\Theta} / \mathrm{Pas} \\
\gamma_{\delta} / \mathrm{Pas} \\
\gamma_{\xi} / \mathrm{Pas} \mathrm{m}^{-1}\end{array}\end{array}$ & $\begin{array}{ll}.03 & \\
& \\
& \end{array}$ & 4 & $\begin{array}{l}\gamma_{\mathrm{eff}}=\gamma_{\Phi} \sin ^{2} \theta=26 \mathrm{mPa} \mathrm{s} \\
\text { Measurement: } 23 \mathrm{mPa} \mathrm{s}\end{array}$ \\
\hline $\begin{array}{c}\text { Electric constants: } \\
\qquad \begin{array}{c}P_{0} / m C m^{-2} \\
\Delta \epsilon_{r}\end{array}\end{array}$ & \multicolumn{2}{|c|}{-1.9} & $\begin{array}{l}\Rightarrow P_{\mathrm{s}}=-45 \mathrm{nC} \mathrm{cm}^{-2} \text { at } \theta=13.8^{\circ} \\
\text { Measurement: } \mathrm{P}_{\mathrm{s}}=-43 \mathrm{nC} \mathrm{cm} \\
\Delta \epsilon_{\mathrm{r}} \approx 300 \text { (22) }\end{array}$ \\
\hline
\end{tabular}

with the uniaxial approximation

$$
\Delta n\left(\vartheta_{s}\right)=n_{0}\left(\frac{n_{e}}{\sqrt{n_{0}^{2} \cos ^{2} \vartheta_{s}+n_{e}^{2} \sin ^{2} \vartheta_{s}}}-1\right)
$$

$\varphi_{0}$ represents the position of the cell with respect to the polarizer for the transmission experiment, $\lambda_{0}$ the wavelength used, $d$ the cell thickness and $n_{0}, n_{e}$ the ordinary and extraordinary refractive indices of the FLC slab.

A comparison of experimentally determined and calculated transmission of the cell before and after the completed high field treatment is represented in Figures 9(a) and 9(d), respectively, and shows a very good agreement especially for the transmission curves after completed high field treatment (Figure 9(d)). The important electrooptical parameters as contrast, switching times, threshold behaviour as well as the optical overshoot are excellently described.

In the polarizing microscope, the intermediate states observed at uncompleted high field treatment, are appearing as heterogeneous arrangements, consisting of 

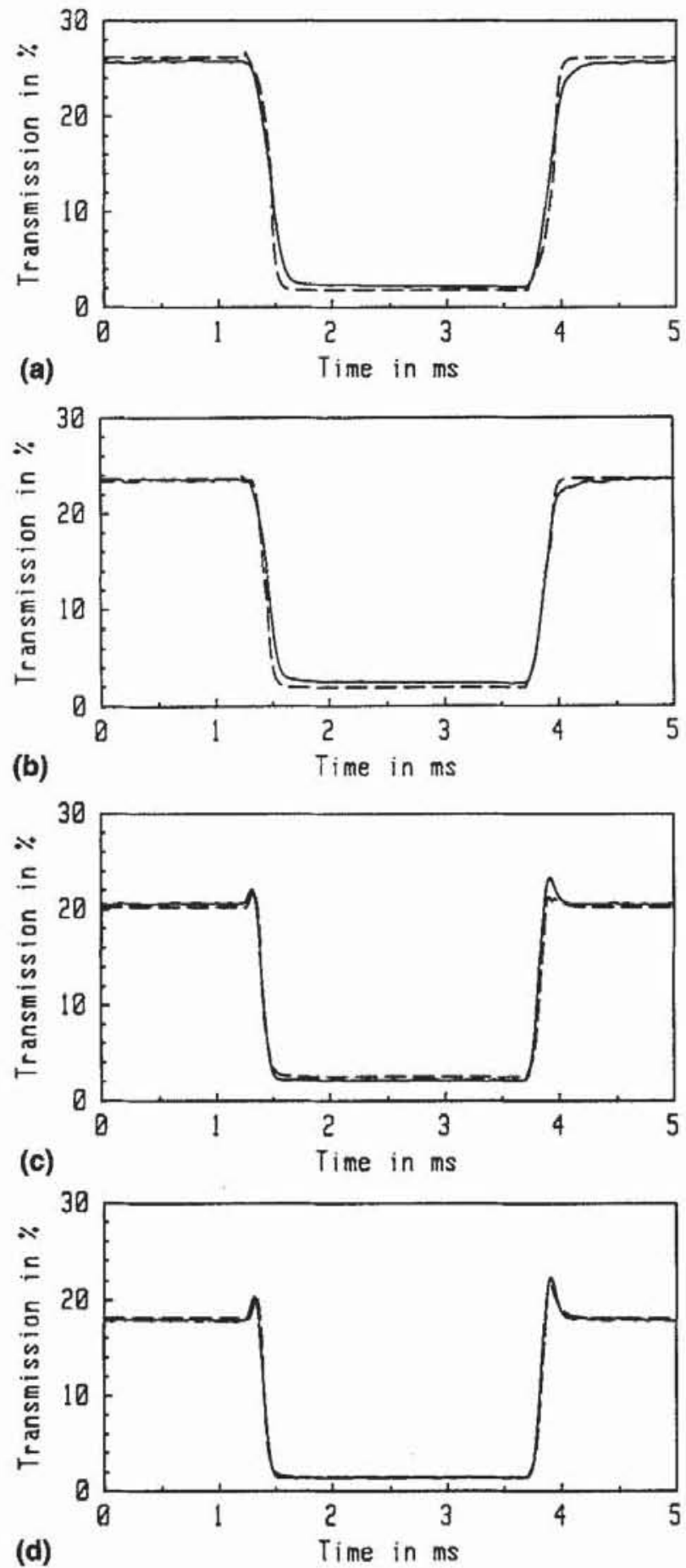

FIGURE 9 Comparison between measured (solid lines) and calculated (dashed lines) transmission curves after (a) $0 \mathrm{sec}$, (b) $2 \mathrm{sec}$, (c) $5 \mathrm{sec}$ and (d) $20 \mathrm{sec}$ time of electrical high field treatment. The calculated curves in (a) and (d) are solutions of the proposed model. The curves in (b) and (c) are combinations of these solutions according to Equation 18. 
areas with the initial layer tilted structure and areas of the finally obtained bookshelf structure, which are separated by a lot of defects (see Section 4.2). According to these observations a quantitative approach to the transmission curves at uncompleted high field treatment can be achieved by a linear combination of the transmissions of the bookshelf and the layer tilted structure and a correction corresponding to the defects' transmission added:

$$
T_{l t / b s}(t)=\chi_{b s} T_{b s}(t)+\left(1-\chi_{b s}\right) T_{l t}(t)+T_{\text {defect }}
$$

$T_{\text {lt } b s}(t)$ is the transmission at time $t$ of the cell at uncompleted high field treatment (cf. Figures 9(b) and 9(c)), $T_{b s}(t)$ the transmission at time $t$ of the cell with pure bookshelf structure obtained after completed high field treatment (cf. Figure 9(d)), $T_{l t}(t)$ the initial transmission at time $t$ of the cell with pure layer tilted structure (cf. Figure 9(a)), $\chi_{b s}$ the share of the bookshelf structure to the total area observed, $T_{\text {defect }}$ the transmission of the defects which are occurring at incompleted high field treatment. $\chi_{b s}$ and $T_{\text {defect }}$ are adjustable parameters and are determined by a least square method. Explicitly, $2 \mathrm{sec}$ of high field treatment (Figure $9(\mathrm{~b})$ ) leads to $\chi_{b s}$ $=35 \%$ and $T_{\text {defect }}=0.3 \%, 5 \mathrm{sec}$ high field treatment (Figure $9(\mathrm{c})$ ) to $\mathrm{X}_{b s}=86 \%$ and $T_{\text {defect }}=1.0 \%$ and the calculated curves agree well with the experimental ones.

Overall, the proposed model describes the electrooptical switching process quite well. The difference of electrooptical behaviour between the two basic structures is expressed by assuming a reversible layer reorientation in the initial chevron structure by setting $\gamma_{\delta}=30 \mathrm{mPa} \mathrm{s}^{-1}$ (Table I) and in the finally obtained bookshelf geometry $(\delta \approx 0)$ by $\gamma_{\delta} \rightarrow \infty$ (Table I), which leads to a fixed layer orientation.

These differences in the reorientational behaviour may be interpretated as an irreversible change of the anchoring conditions which is induced by the high field treatment. In the initial layer tilted structure the anchoring seems to be determined primarily by an interaction between the surface and the molecules with $K_{\text {pre }}=20$ $\mathrm{kN} \mathrm{m}^{-2}$ (Table I) leading to a more or less fixed off-plane angle, which is the angle between the long molecular axis and the surface. Therefore, the necessity of performing a reorientation along the $S_{C}^{*}$ tilt cone at constant off-plane angle involves a coupled reorientation of the smectic layers. The dynamics of this layer reorientation is characterized by a viscous damping constant $\gamma_{\delta}=30 \mathrm{mPa} \mathrm{s}^{-1}$ (Table I). After the high field treatment the initial anchoring breaks down $\left(K_{\text {pre }}=0\right.$, Table I) and now, the anchoring seems to be determined primarily by an interaction between the surface and the smectic layers, which leads to a fixed layer structure at $\delta \approx 0$ (bookshelf geometry). The molecules perform a reorientation along the $\mathrm{S}_{\mathrm{C}}^{*}$ tilt cone at fixed layers, which also leads to a tilt cone reorientation of the macroscopically observable optical axis in the laboratory coordinate system. The fixation of the smectic layer orientation after the high field treatment can be introduced into the model with $\gamma_{\delta} \rightarrow \infty$ (Table I).

All other material constants fitted are not changed by the high field treatment and are comparable with values determined by other methods (Table I). X-ray measurements result in a tilt angle $\theta_{0}$ of $14^{\circ}$ at $40^{\circ} \mathrm{C}$. The fitted Landau coefficients $a_{1}, a_{2}$ and $a_{3}$ lead to $\theta_{0}\left(40^{\circ} \mathrm{C}\right)=13.8^{\circ}$. The effective internal viscosity of rotation along the 
azimuth angle $\gamma_{\Phi, \text { eff }}$ obtained by the field dependence of the reciprocal switching time amounts to $23 \mathrm{mPa} \mathrm{s}^{-1}$ and compares with a fitted value of $26 \mathrm{mPa} \mathrm{s}^{-1}$ with $\gamma_{\Phi, \text { eff }}=$ $\gamma_{\Phi} \sin ^{2} \theta_{0} ; \theta_{0}=13.8^{\circ}$. The integration of the polarization reversal current gives a spontaneous polarization $P_{s}$ of $-43 \mathrm{nC} \mathrm{cm}^{-2}$, the calculated value from the proposed model leads to $-45 \mathrm{nC} \mathrm{cm}^{-2}$ with $P_{s}=P_{0} \sin \theta_{0}\left(\theta=13.8^{\circ}\right)$. The fit of the splay elastic constant results in $K_{s}=160 \mathrm{pN}$ and $K_{s \text {,eff }}=\theta_{0}^{2} K_{s}=9.1 \mathrm{pN}$, which is in the range of values $5 \mathrm{pN}<K_{s, \text { eff }}<50 \mathrm{pN}$ reported by Pelzl, Schiller and Demus ${ }^{21}$ for nonchiral $S_{C}$ phases. The fitted value for the dielectric anisotropy $\Delta \varepsilon_{r}=400$ is in the same order of magnitude as the value of $\Delta \varepsilon_{r}=300$ reported by Gouda et al. ${ }^{22}$ for another $\mathrm{S}_{\mathrm{C}}^{*}$ compound with comparable spontaneous polarization $\left(30 \mathrm{nC} \mathrm{cm}^{-2}\right)$. The value from Gouda et al..$^{22}$ was measured by dielectric spectroscopy $5^{\circ} \mathrm{C}$ below the $\mathrm{S}_{\mathrm{A}}$ $\rightarrow \mathrm{S}_{\mathrm{C}}^{*}$ phase transition, including Goldstone mode contributions.

The large value of the damping constant $\gamma_{\xi}$ for the propagation of the splay deformation agrees with the long relaxation time discussed in 4.1. for the optically uniform $\left(\xi_{t}+\xi_{c}+\xi_{b} \ll d\right)$ into the nonuniform director configuration $\left(\xi_{t}+\xi_{c}+\right.$ $\xi_{b} \approx d$ ) without an electric field. The relaxation time can be estimated by application of Equations (12d) and (2) at $E=0$ :

$$
\gamma_{\xi} \frac{d \xi_{t}}{d t}=\frac{K_{s} \Theta_{0}^{2}}{2 d} \frac{\left(\Phi_{0}-\Phi\right)^{2}}{\xi_{t}^{2}}
$$

Solution of this equation by separation of the variables and integration assuming $\Phi=$ const. $=\pi / 2$ within the limits $\xi(t=0)=\xi_{0}$ and $\xi\left(t=t_{\text {relax }}\right)=d / 4$ leads with the approximation $0 \approx \xi_{0}^{3} \ll(d / 4)^{3}$ to

$$
t_{\text {relax }}=\frac{d^{4} \gamma_{\xi}}{24 \pi^{2} K_{s} \theta_{0}^{2}}
$$

and $t_{\text {relax }}=2.1 \mathrm{sec}$ for the system considered $\left(d=3.9 \mu \mathrm{m}, \gamma_{\xi}=20 \cdot 10^{12} \mathrm{~Pa} \mathrm{~s}\right.$ $\mathrm{m}^{-1}, \Theta_{0}=13.8^{\circ}$ and $K_{s}=160 \mathrm{pN}$ ). An experimental value of $2 \mathrm{sec}$ (Section 4.1) was obtained, which agrees excellently with the value calculated from the fitted constants.

The solution of the Equations of motion (12a-f) leads consequently to the dynamics of the director reorientation in the $S_{C}^{*}$ coordinate system that is the time dependence of the variables $\Phi, \theta, \delta, \xi_{t}, \xi_{c}$ and $\xi_{b}$. Figures $10(a)$ and $10(\mathrm{~b})$ depict the time dependent thickness of the nonuniform boundary layers and the chevron interface. The symmetric boundary conditions lead to $\xi_{t}(t)=\xi_{b}(t)$ with $\Phi_{0}=0$. The nonuniform layers are summed up to $10 \%$ at the most of the total cell thickness and each nonuniform layer is much smaller than the wavelength of light used (546 $\mathrm{nm}$ ) which means, that the system can be described as optically uniform. The changes in thickness of these layers are very small, compared to the total thickness of the cell.

The time dependence of the layer tilt angle $\delta$ (Figure 11) confirms the interpretation of the switching procedure of Section 4.2. In the layer tilted geometry before the high field treatment a reversible layer reorientation takes place, which com- 

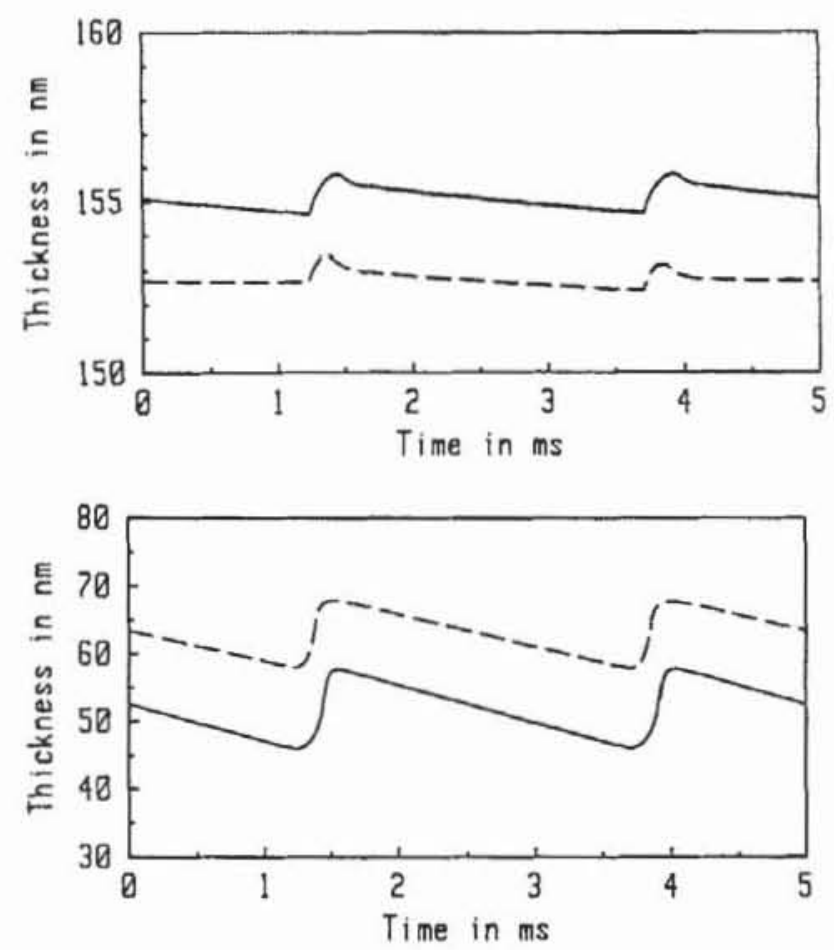

FIGURE 10 Thickness $\xi_{b}, \xi_{b}$ and $\xi_{c}$ of nonuniform layers vs. time before (solid lines) and after completed (dashed lines) electrical high field treatment resulting from a fit of the proposed model to the experimental data. (a) top and bottom boundary layer $\left(\xi_{t}\right.$ and $\left.\xi_{b}\right)$ and (b) chevron interface $\left(\xi_{c}\right)$.

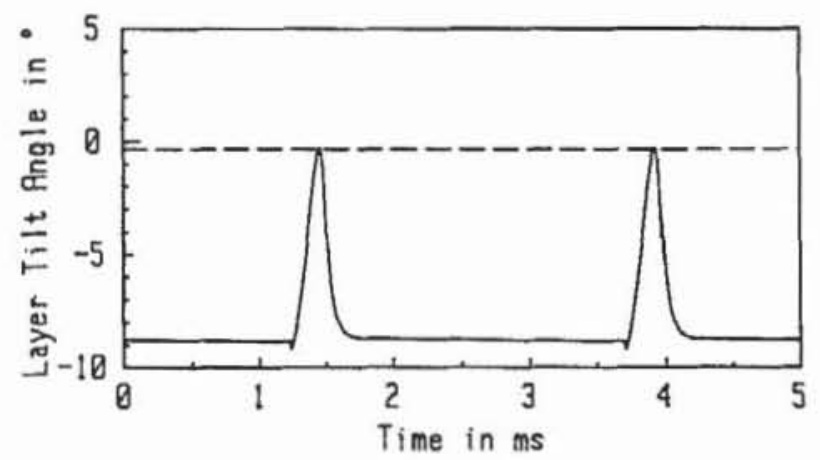

FIGURE 11 Smectic layer tilt angle $\delta$ vs. time before (solid line) and after completed (dashed line) electrical high field treatment resulting from a fit of the proposed model to the experimental data. The strong variations before the high field treatment are indicating the reversible layer reorientation. After the high field treatment the layers are fixed at $\delta \approx 0$.

pensates the off-plane motion of the director. After the high field treatment a bookshelf geometry with fixed layers $(\delta(t)=0=$ const.) is present.

The tilt angle $\Theta$ changes in time from $12^{\circ}$ to $16^{\circ}$ and points towards a large electroclinic effect (Figure 12). Especially at the beginning of the switching, a drop of the tilt angle is observed as was predicted by Andersson et al. ${ }^{18}$ The reorientation dynamics of the azimuth angle $\Phi$ (Figure 13) is scarcely affected by the high field treatment.

In conclusion the proposed model, for which a layer reorientation was introduced, describes consistently the dynamics of the electrooptical behaviour of the system and its changes during a high field treatment. In addition, the model also describes 


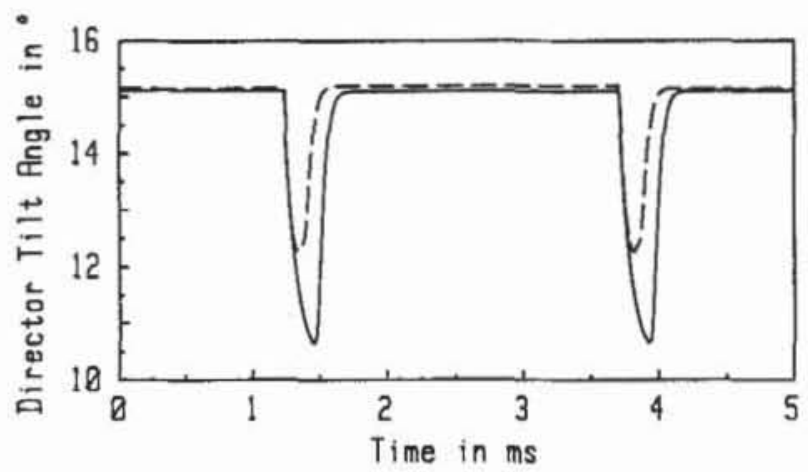

FIGURE 12 Director tilt angle $\Theta$ vs. time before (solid line) and after completed (dashed line) electrical high field treatment resulting from a fit of the proposed model to the experimental data.

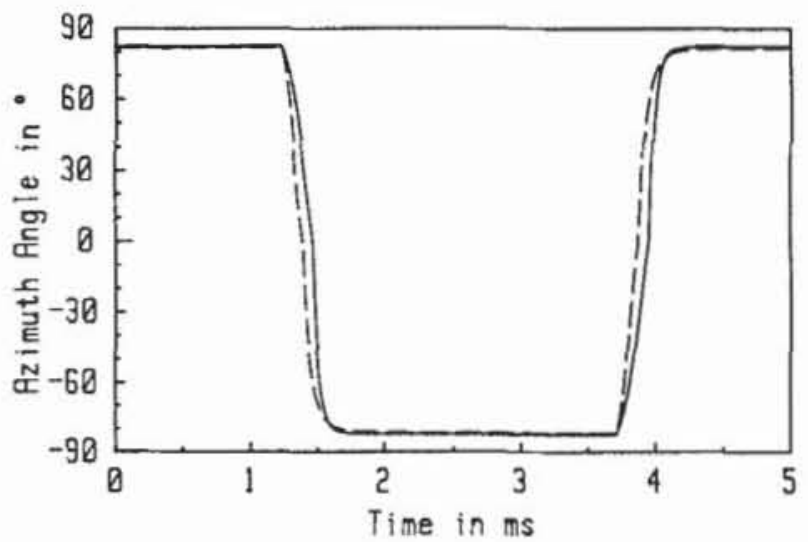

FIGURE 13 Azimuth angle $\Phi$ vs. time before (solid line) and after completed (dashed line) electrical high field treatment resulting from a fit of the proposed model to the experimental data.

the field, frequency, amplitude and temperature behaviour of the system consistently, as will be reported in a separate paper. ${ }^{23}$

\subsection{Layer Reorganization}

A reversible layer reorientation and a layer upraising have been introduced by the proposed model for a description of the optical behaviour. A mechanism of such motions cannot be given at the present but some general ideas may be exposed.

An elastic deformation of smectic layers seems unlikely, since it needs a change in smectic layer thickness which requires more energy than is available by a $P$, $\vec{E}$ interaction and an additional term due to the elastic bend deformation of the layers has to be introduced in the potential density expansion (Equation 2). The model presented requires no such term.

A model with a transfer of defects does not exhibit these difficulties. A shift of defects may convert easily a planar tilted or chevron gcometry into a bookshelf geometry as demonstrated in Figure 14 . Such a process may better be referred to as layer reorganization than layer reorientation and exhibits advantages in the interpretation of the results.

A reorganization of smectic layers needs solely high activation energy which is present in the highly deformed layer structure within the defects and an additional 
(a)

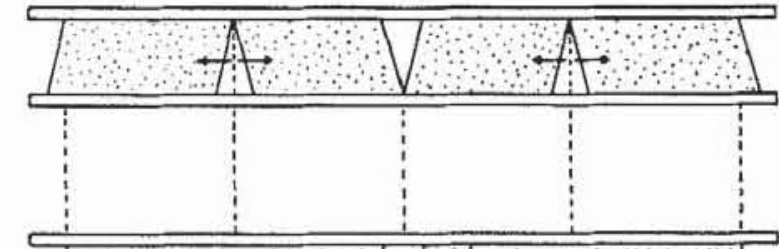

(b)

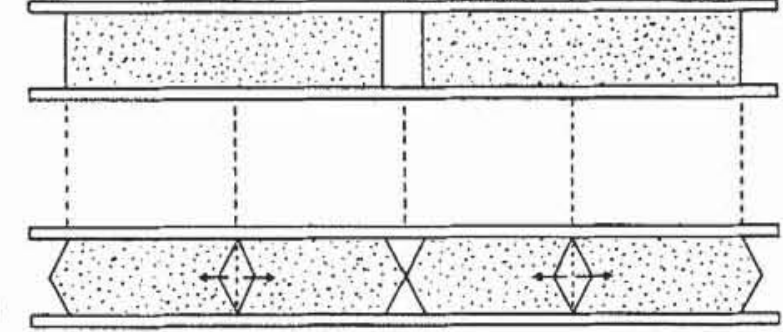

FIGURE 14 Possible model for a transformation of a layer tilted (a) and a chevron (c) structure into a bookshelf structure (b) by a shift of defects.

bending term in the potential density expansion is not needed. In this context, the soliton description of the chevron structure may be relevant. ${ }^{24} \mathrm{~A}$ field induced reversible motion of zig-zag defects was reported and related to a change in the chevron structure. ${ }^{25}$ However, a reversible layer reorientation was clearly established by X-ray experiments, ${ }^{14,15}$ at least for compounds with high spontaneous polarization, and further experiments are necessary for an understanding of these reorientations.

\section{CONCLUSIONS}

The model of an optically uniform director reorientation with a coupled layer reorganization may be considered as the limiting case in which the $\bar{P}, \vec{E}$ interaction dominates elastic interaction. In contrast, if the elastic interaction dominates all other interactions in the cell, a nonuniform director reorientation with fixed layers is found ${ }^{5,6}$ representing another borderline case.

The deviations of the mean director motion from a uniform reorientation along the $\mathrm{S}_{\mathrm{C}}^{*}$ tilt cone observed in the laboratory coordinate system is caused by an additional reversible motion of the smectic layers. This motion is suppressed if the layers are irreversible upraised and anchored at high electric field and the expected director reorientation along the $S_{C}^{*}$ tilt cone is then observed in the laboratory coordinate system.

A potential density expansion describes the coupled director-layer reorientation and leads to a simultaneous determination of a number of material and cell constants as fitted parameters and to a dymanic model of SSFLC cells.

\section{Acknowledgments}

This work was supported by a grant from Deutsche Foschungsgemeinschaft. Discussions with G. Pelzl, P. Schiller, Martin-Luther-Universität, Halle-Wittenberg and J. E. Maclennan, Universität Mainz, are gratefully acknowledged. 


\section{References}

1. R. B. Meyer, L. Liebert, L. Strzelecki and P. Keller, J. de Phys. Lett., 36, L-69 (1975).

2. R. B. Meyer, Mol. Cryst. Liq. Cryst., 40, 33 (1977).

3. N. A. Clark and S. T. Lagerwall, Appl. Phys. Lett., 36, 899 (1980).

4. J. E. Maclennan, N. A. Clark, M. A. Handschy and M. R. Meadows, Liq. Cryst., 7, 753 (1990).

5. J. E. Maclennan, M. A. Handschy and N. A. Clark, Liq. Cryst. 7, 787 (1990) and Zhiming Zhuang, N. A. Clark and J. E. Maclennan, Liq. Cryst. 10, 409 (1991).

6. I. Abdulhalim, G. Moddel and N. A. Clark, Appl. Phys. Lett., 60, 551 (1992).

7. F. Gießelmann and P. Zugenmaier, Liq. Cryst., 8, 361 (1990).

8. F. Gießelmann and P. Zugenmaier, Proceedings of the 20th Freiburger Arbeitstagung Flüssigkristalle, Freiburg (FRG) 1991.

9. J. S. Patel, Sin-Doo Lee and J. W. Goodby, Phys. Rev. A, 40, 2854 (1989).

10. G. Andersson, I. Dahl, L. Komitov, S. T. Lagerwall, M. Matuszczyk and K. Skarp, 13th International Liquid Crystal Conference, Vancouver, Canada 1990 (Book of Abstracts II-168).

11. W. J. A. M. Hartmann, G. Vertogen, C. J. Gerritsma, H. A. v. Sprang and A. G. H. Verhulst, Europhys. Lett., 10, 657 (1989).

12. W. J. A. M. Hartmann, G. Vertogen, C. J. Gerritsma, H. A. v. Sprang and A. G. H. Verhulst, Ferroelectrics, 113, 257 (1991).

13. M. Johno, A. D. L. Chandani, Y. Ouchi, H. Takezoe, A. Fukuda, M. Ichihashi and K. Furukawa, Jpn. J. Appl. Phys. Lett., 28, L-119 (1989).

14. K. Itoh, M. Johno, Y. Takanishi, Y. Ouchi, H. Takezoe and A. Fukuda, Jpn. J. Appl. Phys., 30, 735 (1991).

15. M. Oh-E, M. Isogai and T. Kitamura, Liq. Cryst., 11, 101 (1992).

16. K. Mohr, S. Köhler, K. Worm, G. Pelzl, S. Diele, H. Zaschke, D. Demus, G. Andersson, I. Dahl, S. T. Lagerwall, K. Skarp and B. Stebler, Mol. Cryst. Liq. Cryst., 146, 151 (1987).

17. K. H. Yang, J. Appl. Phys., 64, 4780 (1988).

18. G. Andersson, I. Dahl, W. Kuczynski, S. T. Lagerwall, K. Skarp and B. Stebler, Ferroelectrics, 84, 285 (1988).

19. H. Takezoe, Y. Ouchi, K. Ishikawa and A. Fukuda, Mol. Cryst. Liq. Cryst., 139, 27 (1988).

20. I. Dahl, S. T. Lagerwall and K. Skarp, Phys. Rev. A, 36, 4380 (1987).

21. G. Pelzl, P. Schiller and D. Demus, Liq. Cryst., 2, 131 (1987)

22. F. Gouda, K. Skarp and S. T. Lagerwall, Mol. Cryst. Liq. Cryst., 209, 99 (1991).

23. F. Gießelmann and P. Zugenmaier, Liq. Cryst., in press.

24. M. Nakagawa, Mol. Cryst. Liq. Cryst., 174, 65 (1989).

25. A. Fukuda, Y. Ouchi, H. Arai, H. Takano, K. Ishikawa and H. Takezoe, Liq. Cryst., 5, 1055 (1989). 